\title{
ETNOGRAFÍA A LO BRUTO: Un opening de datos muy salvaje
}

\section{María Fernanda MOSCOSO}

Flacso-España moscoso.r.maria@gmail.com

\section{ROUGH ETHNOGRAPHY: A wild data opening}

Resumen: El artículo que se presenta tiene como fin introducir una serie de problemáticas (¿dónde empieza lo bruto?, ¿el hacer que se distingue del pensar?, ¿existe un observador separado de la realidad?, ¿se pueden pensar los archivos fuera de los contextos?) que nacieron a raíz de la apertura de los archivos etnográficos en el marco del seminario-taller "Etnografía a lo bruto. Un en de datos muy salvaje". La propuesta de exponer los archivos etnográficos (vinculados a procesos de aprendizaje) y de abrir un diálogo tuvo lugar partiendo de una -primera- premisa: los archivos etnográficos son un conjunto de datos (notas, diarios, videos, fotografías, grabaciones, entrevistas, observaciones u objetos) que han sido recolectados durante el trabajo de campo y que no han pasado por un proceso de sistematización. Se trata de una reflexión que, desde una perspectiva metodológica, se ocupa de pensar la investigación etnográfica en dos niveles: por una parte, nos proponemos pensar de qué hablamos cuando nos referimos a los procesos de investigación etnográfica (sobre el aprendizaje y la educación) inacabados o en ciernes y qué se puede pensar a partir del gesto de abrir los archivos.

Abstract: The present article aims at introducing some issues arisen from the opening of ethnographic archives in the seminar-work „Rough ethnography: a wild data opening”. The proposal to expose the ethnographic archives (linked to learning processes) and to open a dialogue started from a -first- premise: ethnographic files constitute a set of data (notes, journals, videos, photographs, recordings, interviews, observations or objects) that have been collected during the fieldwork and have not gone through a process of systematization. It is a reflection that, from a methodological perspective, deals with thinking ethnographic research at two levels: on the one hand, we intend to think about what we mean when we refer to unfinished or budding processes of ethnographic research (on learning and education), on the other hand what can be thought from gesture of opening the files.

Palabras clave: Etnografía. Archivos. Metodologías experimentales. Procesos de aprendizaje Ethnography. Archives. Experimental methodologies. Learning processes 


\title{
Abrir el archivo etnográfico (o la falta de arsénico)
}

\begin{abstract}
"Ahora bien, ¿qué pasa si no me limito a visitar el almacén sino que trato de meter mi cuerpo en su interior y al mismo tiempo permito que el almacén entre en mi cuerpo?....entonces el archivo se hará visible a través de mi cuerpo" (Nachbar, 2008).
\end{abstract}

Los científicos y los militares no reflexionan sobre su hacer porque cambia el hacer, señala Maturana ${ }^{1}$. En otras palabras, si te preguntas por los fundamentos del hacer, cambia el hacer. Y si el hacer cambia, todo se transforma, incluidos los conocimientos y los cuerpos que los producen. La certidumbre niega la reflexión.

Imaginemos un seminario de metodología de la investigación: las sillas y las mesas organizadas en un aula dispuesta para que los futuros antropólogos aprendan cosas como qué es investigar, quién investiga, por qué vamos al campo, qué conocimientos son útiles y qué se debería olvidar. El aprendizaje del oficio etnográfico está basado pues, en ciertos principios o puntos de partida que parecen preexistentes y en consecuencia, incuestionables. Porque qué es investigar, por qué vamos al campo y cuáles son los conocimientos útiles son cuestiones que implican una serie de ideas y nociones que son epistemológicas, políticas y éticas. Ideas y nociones que, por cierto, no siempre suelen ser objeto de revisión.

Los seminarios de metodología de la investigación suelen estar centrados en el aprendizaje de una serie de herramientas, teorías y técnicas con los que los profanos han de ir al campo de trabajo a recolectar información. Cuatro ideas al respecto: Uno. Ir al campo se asocia al deseo de entrar en contacto con el "otro" y más aún, de interpretar los fenómenos sociales viendo desde "dentro" (lo EMIC). Dos. No hace falta leer los diarios de campo de Malinowski para considerar la idea de que, desde sus inicios, la investigación es experimentada como un proceso individual. Pero siempre merece la pena echar un vistazo para no olvidarlo:

"Último día del mes, completo colapso. Por la mañana no hice nada. Por la tarde, la mina de Dobu hizo su llegada; tomé fotos de la canoa y hablé con el policía de Saranoa. Subjetivamente: una situación general en la que necesito narcóticos (...) Terminé hacia las 12, y me sentía tan quebrantado y somnoliento (¿falta de arsénico?)“ (Malinowski: 1967: 249).

Tres. La investigación etnográfica está centrada en el producto y no en el proceso. Es cada vez más común que la maquinaria académica exija unos resultados que son valorados en función del cumplimiento de una serie de estándares y formatos: publicación en masa en revistas indexadas, asistencia a congresos, etc. Cuatro. El material recolectado durante el período de trabajo de campo es un archivo cerrado. El cuaderno de campo, por ejemplo, es una suerte de prolongación del diario personal: el lugar recóndito donde el observador escribe, desde su intimidad, sus apreciaciones e ideas sobre las personas y el lugar en el que se encuentra.

"Etnografía a lo bruto. Un opening de datos muy salvaje" es un seminario-taller experimental que surge con el fin de abordar estas problemáticas en la medida en la que propone abrir un espacio de investigación colectivo, centrado en el proceso y cuyo fin es abrir los archivos etnográficos y discutirlos públicamente.

La pulsión de archivar se acompaña también por la pulsión de abrir los archivos, mostrar-

1 Entrevista realizada a Humberto Maturana en el programa „La belleza del Pensar”. 
los, exponerlos. Y el gesto de abrir los archivos sólo puede entenderse si se parte de la premisa de que existe algo que está cerrado, es decir, que no tiene conexión con el exterior. Y que, por lo tanto, puede ser objeto de uno o varios agujeros a través de los cuales pasa la luz, es decir, de una apertura. El artículo que se presenta tiene como fin introducir una serie de preguntas que nacieron a raíz de la apertura de los archivos etnográficos ${ }^{2}$ en el marco del seminario-taller "Etnografía a lo bruto. Un Opening de datos muy salvaje" que tuvo lugar en otoño del $2014^{3}$ y en el 2015. La propuesta de exponer los archivos etnográficos (vinculados a procesos de aprendizaje) y de abrir un diálogo tuvo lugar partiendo de una -primera- premisa: los archivos etnográficos son un conjunto de datos (notas, diarios, videos, fotografías, grabaciones, entrevistas, observaciones u objetos) que han sido recolectados durante el trabajo de campo y que no han pasado por un proceso de sistematización. Se trata de material salvaje. A lo largo del taller, tuvo lugar una reflexión que, desde una perspectiva metodológica, se ocupó de pensar la investigación etnográfica en dos niveles: por una parte, nos propusimos pensar de qué hablamos cuando nos referimos a los procesos de investigación etnográfica (sobre el aprendizaje y la educación) inacabados o en ciernes. Esto es, sobre una etapa muy concreta del proceso de investigación que las prácticas académicas, obsesionadas por la presentación de unos resultados que se asumen asépticos y objetivos, suelen borrar. Y, por otra parte, invitamos a que la gente traiga su material etnográfico en bruto, lo exponga, lo muestre y lo comparta con el fin de pensar en análisis e interpretaciones probables e improbables. A continuación, se expondrán de modo sucinto algunas de las preguntas y planteamientos que surgieron de las discusiones y reflexiones que se hicieron colectivamente ${ }^{4}$. Se trata, en consecuencia de ideas sencillas que tienen un carácter introductorio y que se presentan como interrogantes. Las breves reflexiones que las acompañan representan, en este sentido, un intento por ilustrar las preguntas.

\section{Dónde empieza "lo bruto"}

¿Realmente es sostenible que los archivos etnográficos son un conjunto de datos que no han pasado por un proceso de sistematización? La idea de que los datos son salvajes se sustenta en concepciones muy concretas sobre la etnografía. Etimológicamente, la etnografía viene de la palabra griega "ethnos" que significa pueblo y "grapho" que significa, por una parte, "escribo" y, por la otra "descripción del pueblo". De modo mayoritario, suele primar la idea de que la etnografía es una suerte de descripción del mundo. Se puede señalar al menos dos cuestiones al respecto: en primer lugar, que llevar a cabo historias de vida o escribir un diario de campo, por ejemplo, no representa simplemente poner en práctica, de modo automático, un conjunto de técnicas antropológicas. Hacer una entrevista auto-biográfica implica poner en práctica determinadas ideas sobre el tiempo pues se asumen que las personas forman parte de trayectorias de vida que pueden ser descritas a través de la producción de material discursivo. En segundo lugar, la idea de que los datos que se recolectan durante el trabajo de campo son inmaculados también es sospechosa. Merece la pena preguntarse si la aplicación de una serie de técnicas es ascéptica y no afecta directamente la información que se recoje. Es necesario, de esta manera, preguntarse dónde empieza y dónde termina lo „bruto" y por qué tendemos a pensar que describir el mundo no representa intervenir sobre él.

\footnotetext{
2 Se trata de un espacio colectivo /// de investigación etnográfica/// sobre aprendizajes y educación, organizado junto a David Poveda, del Grupo de Etnografía y Educación del IMA.

3 En INTERMEDIAE-MATADERO (Madrid). Una segunda parte del seminario tiene lugar de diciembre 2015 a febrero del 2016.

4 Agradecimientos especiales a Selina Blasco, Alejandro Simón, Marta Morgade, Susana Moliner, Suraia Abud, Karmele Mendoza, Viviana Silva, Adolfo Estalella, Tomás Sánchez-Criado, Cristian Rozas, Ana María Relaño Pastor, Cristina Carrillo y Manuel Palomares. Y, por supuesto, a todo el maravilloso equipo de Intermediae.
} 


\section{El hacer que se separa del pensar}

A lo mejor resulta interesante detenerse en la idea de la fase de producción de los archivos etnográficos. Se ha dado por hecho que en la investigación antropológica hay al menos dos momentos: el trabajo de campo y, después, la sistematización e interpretación de los datos. Durante el trabajo de campo se recogería la información que conformaría lo que aquí hemos llamado los archivos etnográficos. La práctica y la teoría. Pero ¿dónde reside esta división?, ¿cuáles son las líneas que distinguen el hacer y el pensar? Mientras el discurso hegemónico-académico prefiere sostener que, efectivamente, existen divisiones entre el hacer y el teorizar (y en ninguno de los momentos está el cuerpo), la crítica de-colonial sobre la producción del pensamiento se puede extender al campo mencionado pues, como se ha señalado, la separación entre dos procesos (el trabajo de campo y la teoria) forma parte de una matriz colonial de producción de conocimientos que necesita clasificar los momentos de la investigación, como si éstos no fuesen profundamente mestizos e incluso, ambiguos. Los sujetos, sus prácticas, creencias o puntos de vista sobre el mundo han sido clasicamente la materia prima que utiliza quien posee los conocimientos teóricos y metodológicos para trabajar la materia. El otro no produce teoría, no es agente del conocimiento ni posee una ontología. El vínculo entre quien estudia y quien es estudiado reproduce estructuras desiguales -no solamente de género, etnicidad, clase social o edad- también de conocimientos. En este sentido, si bien es cada vez más común el esfuerzo por llevar a cabo etnografías participativas cuyo fin es resquebrajar las dinámicas en las que el otro se queda fuera, ¿cuál es el sentido de continuar separando los momentos de la investigación?

\section{¿Existe un observador separado de la realidad?}

El mundo del "afuera" y lo que hago para estar "en ese mundo" son inseparables. Esta perspectiva de la mente como enactivamente encarnada tiene dos consecuencias ya que, si la mente no está en la cabeza, ¿dónde diablos está? se pregunta Varela (2000: 242.) Este es precisamente el punto: es en este no-lugar de la co-determinación entre lo interno y lo externo, luego no podemos decir que está afuera o adentro. No existe un observador separado de la realidad pues la mente es inseparable del organismo como un todo que es afectado al tiempo que afecta la realidad. No sólo estamos dotados de un cerebro, sino de todo un cuerpo. Aquí, la metáfora de la mirada es especialmente útil con el fin de ilustrar que los ojos han sido utilizados para resignificar una perversa capacidad, refinada hasta la perfección en la historia de la ciencia -relacionada con el militarismo, el capitalismo y el colonialismopara distanciar el sujeto conocedor de todos y de todo en interés del poder sin trabas. Los instrumentos de visualización han compuesto esos significados de des-encarnación (Haraway 1995). Pero el cuerpo no es independiente del mundo, sino que, por medio de la percepción, el cuerpo y el mundo permanecen entrecruzados. Una de las consecuencias principales (Merleau-Ponty 2000) es que la percepción adquiere una connotación epistemológica, en torno a la cual gira el problema de la verdad del subjetivismo y el objetivismo, categorías que es imposible asumir sin más debido a las implicaciones corporales con que se ven condicionadas, "ya que la percepción no es una ciencia del mundo sino el trasfondo sobre el que se destacan todos los actos y que todos los actos presupone“ (González 2011). Con la percepción fundimos estos términos en una experiencia que encarna toda vinculación con el mundo, al que nuestro cuerpo pertenece, naturalmente. Poner el cuerpo en la investigación es, por lo tanto, un reto epistemológico y político y afecta directamente la idea de observación etnográfica.

\section{¿Se pueden pensar los archivos fuera de los contextos?}

Como se ha señalado, suele entenderse que la etnografía es una descripción: la acumu- 
lación de datos y experiencias que son asumidos como el contexto de las investigaciones (Blommaert 2006). Sin embargo, no es lo mismo describir los contextos de las investigaciones que pensar en términos de conocimientos situados. A lo mejor es posible preguntarse, en este sentido, si abrir los archivos no implica también abrir los contextos, esto es, visibilizarlos. Y aquí, abrir los contextos de producción de datos etnográficos equivaldría a no descartar las condiciones de producción de estos datos desde al menos dos puntos de vista. En primer lugar, habría que pensar que el material "en bruto" es tanto un producto de situaciones sociales como unas prácticas situadas social e históricamente (García, 2000; Devillard, 2004). En segundo lugar y como consecuencia de lo anterior, abrir el contexto de producción de datos etnográficos también sería una invitación a pensar en la visión desde un cuerpo complejo, contradictorio, estructurante y estructurado contra la visión desde arriba, desde ninguna parte, desde la simpleza (Haraway, 1995). Abrir los contextos, desde una perspectiva feminista, implicaría, en consecuencia, situar la producción de datos etnográficos o, lo que es lo mismo, asumir que existen unos marcos de referencia que están dados y que son los puntos de partida en los que tienen lugar las investigaciones etnográficas, una posición (de clase, edad, género, clase social, ciudadanía, edad) desde la cual se produce el conocimiento y unos sesgos que no siempre son explicitados.

\section{¿Es posible pensar en una práctica etnográfica centrada en el proceso de investigación (y no en los resultados)?}

El oficio de la investigación, en general, está centrado en unos resultados que se caracterizan por ser un producto. Un fruto que se fabrica gracias a un proceso sistemático que ha depurado, ordenado, sistematizado e interpretado un conjunto de datos arrancados de la realidad. Del árbol. Enfocar la atención en los resultados permite eliminar los restos innecesarios los cuales pasan a formar parte del material de archivo, en el mejor de los casos. Esto es así, entre otros motivos, gracias a la identificación entre la así llamada verdad y el método (Maturana 1997) lo cual ha conducido a que el segundo sea el corazón del discurso científico, lo cual, a su vez, ha impactado en la producción de conocimientos. El método existe porque hay un procedimiento que borra las huellas. Pero, ¿y si el error fuese un lugar de aprendizaje? Generalmente, los resultados de una investigación se presentan como un producto aséptico en el que se han borrado las huellas: los errores, los desaciertos, los tachones o los momentos de pérdida o confusión. ¿Y si se decidiera exponer los fallos, remover la basura e incluso volver a los datos que son inservibles? Recuperar el error es a lo mejor un ejercicio pedagógico pues se puede aprender del proceso como un espacio en el que se ensayan, formulan e imaginan estrategias de investigación. En otras palabras, centrar el conocimiento científico en los resultados cierra las posibilidades de aprendizaje pues el deshecho y el error constituyen material pedagógico que da lugar a situaciones de aprendizaje en las que las personas posiblemente somos capaces de generar nuevos conocimientos y herramientas sobre la investigación. Se trataría, como señala Massumi (2002), de la posibilidad de acceder a campos creativos todavía no agotados de posibilidades impalpables.

En resumen, el gesto de abrir los archivos etnográficos ha permitido establecer una serie de preguntas sobre las prácticas etnográficas que son, desde mi perspectiva, muy pertinentes y que aquí se han presentado de modo introductorio. Se ha señalado, en primer lugar, que interrogarnos sobre la intervención que se lleva a cabo al describir la realidad permite señalar que los datos no son necesariamente „salvajes“ o „brutos“ en el sentido de que el trabajo de campo, a través de las técnicas de investigación (entrevista, observación, diario de campo, etc), produce una afectación sobre el mundo. Por otra parte, pensar que el "hacer" se separa del "pensar" supone reproducir unas prácticas coloniales de producción de conocimientos cuyo corazón es un método científico que separa de modo artificial las dinámicas etnográficas: quién piensa y quién es pensado no ocupan el mismo lugar. Si bien 
se trata de una idea que se viene repitiendo cada vez con más frecuencia, la generación de metodologías de-coloniales aún es una labor pendiente por experimentar e imaginar en el mundo académico. Esto se vincula con otra pregunta, la cual gira alrededor del lugar del cuerpo en las investigaciones. Porque poner el cuerpo en el centro de la investigación etnográfica, lejos de alejar al sujeto conocedor del mundo, del mundo, lo funde, transformando nuestras ideas sobre la percepción. En la observación hay unas políticas de la mirada que han de ser deconstruídas: ¿cómo observamos la realidad?, ¿qué herramientas usamos para hacerlo?, ¿cómo afecta nuestro modo de observar sobre aquello que observamos?, ¿cómo incide aquello que observamos sobre la construcción del conocimiento?, ¿cuáles son las implicaciones políticas (de género, clase, ciudadanía o edad) de nuestra mirada? son algunas de las preguntas que develan el papel del cuerpo en las investigaciones, y permiten hablar de las políticas de la mirada.

Por otra parte y en relación con lo anterior, en el ejercicio que se ha llevado a cabo en este artículo, se sostiene que explicitar los locus de enunciación no es ni puede ser solamente una opción ética; se trata también de la producción de conocimientos que tienen lugar en contextos, esto es, desde posiciones que no siempre suelen ser suficientemente explicitadas. Posiciones (de edad, estudios, ciudadanía, género, pertenencia étnica, clase, etc) que, por supuesto, implican imposiciones de diversa índole (categorías, puntos de vista, voces, opiniones) sobre los "otros". El reto sería, desde esta perspectiva, visibilizar las situaciones sociales como unas prácticas situadas social e históricamente y de llevarlo a cabo desde una práctica centrada, además, en el proceso y no únicamente en los resultados. Esto implicaría, por una parte, cuestionar la aceleración neo-liberal del trabajo académico, obsesionado con la producción de resultados en detrimento del pensar lento y de los cuerpos que los producen. Y por otra, de convertir el deshecho y/o el error en una oportunidad para el aprendizaje y no en una huella que ha de ser eliminada/borrada.

\section{Bibliografía}

Bloomaert, Jan (2006). Ethnographic fieldwork: A beginner's guide. Londres: University of London.

Devillard, María José (2004). "Antropología social, enfoque (auto) biográfico y vigilancia epistemológica", Revista Antropología Social, 13: 161-184.

García, José Luis (2000). "Informar y narrar: el análisis de los discursos en la investigaciones de campo", Revista Antropología Social, 9: 75-4.

González, Andrés (2011). "Fenomenología del entrecruce del cuerpo y el mundo en Merlau-Ponty", Ideas y Valores, 145: 113-130.

Haraway, Dora J. (1995). Ciencia, ciborgs y mujeres. La reinvención de la naturaleza. Madrid: Cátedra.

Massumi, Brian (2002). Parables for the Virtual: Movement, Affect, Sensation. Durham: Duke University Press.

Maturana, Humberto (1997). La objetividad; un argumento para obligar. Santiago: Dolmen.

Malinowski, Bronislaw (1989). Diario de campo en Melanesia. Gijón: Júcar.

Merleau-Ponty, Maurice (2000). Fenomenología de la percepción. Barcelona: Península.

Varela, Francisco (2000). El fenómeno de la vida. Santiago: Dolmen. 